\title{
Liquid Biopsy and Tissue Biopsy Comparison with Digital PCR and IHC/FISH for HER2 Amplification Detection in Breast Cancer Patients
}

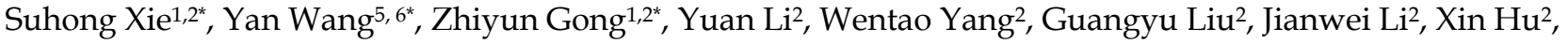

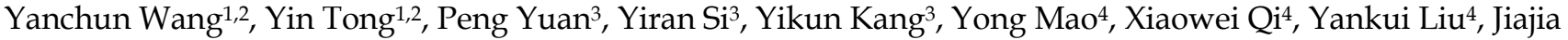 \\ $\mathrm{Ou}^{5}$, Zhaoliang Li5 ${ }^{5}$, Xin Pan ${ }^{5}$, Zhaoqing Lv ${ }^{5}$, Kavanaugh Kaji ${ }^{6}$, Lin Guo ${ }^{1,2}$, Renquan $\mathrm{Lu}^{1,2}{ }^{\bowtie}$ \\ 1. Department of Clinical Laboratory, Fudan University Shanghai Cancer Center, Shanghai, P. R. China. \\ 2. Department of Oncology, Shanghai Medical School, Fudan University, Shanghai, P. R. China. \\ 3. Department of VIP Medical Services, National Cancer Center/National Clinical Research Center for Cancer/Cancer Hospital, Chinese Academy of Medical Sciences \\ and Peking Union Medical College, Beijing, P. R. China. \\ 4. Department of Oncology, Affiliated Hospital of Jiangnan University, Wuxi, P. R. China. \\ 5. Questgenomics, No.12 Mozhou East Rd, Nanjing, Jiangsu, P. R. China. \\ 6. Gnomegen, 6440 Lusk Blvd, D207, San Diego, CA, 92121 United States. \\ *These authors contributed equally to this work.
}

$\triangle$ Corresponding authors: Lin Guo, Department of Clinical Laboratory, Fudan University Shanghai Cancer Center; Department of Oncology, Shanghai Medical School, Fudan University, Shanghai 200032, P. R. China. Phone: (+86) 021-64175590; E-mail: guolin500@hotmail.com; Renquan Lu, Department of Clinical Laboratory, Fudan University Shanghai Cancer Center; Department of Oncology, Shanghai Medical School, Fudan University, Shanghai 200032, P. R. China. Phone: (+86) 021-64175590; E-mail: lurenquan@126.com.

(C) The author(s). This is an open access article distributed under the terms of the Creative Commons Attribution License (https://creativecommons.org/licenses/by/4.0/). See http://ivyspring.com/terms for full terms and conditions.

Received: 2021.08.28; Accepted: 2021.12.02; Published: 2022.01.01

\begin{abstract}
Two hundred twenty-four breast cancer patients with paired tissue and plasma samples were enrolled from 3 clinical centers to evaluate sensitivity and specificity of a digital PCR HER2 amplification assay. All patients were histologically confirmed diagnosis of locally advanced and recurrent or metastatic breast cancer with stage III/IV and had tissue HER2 status determinations using IHC/FISH. For the whole 224 advanced breast cancer patients, the sensitivity between dPCR in plasma and IHC/FISH in tissue samples is $43.75 \%(42 / 96)$, the specificity is $84.38 \%(108 / 128)$ and the overall concordance is $66.96 \%(150 / 224)$. Interestingly, when we looked at stage III, stage IV and recurrent or metastatic breast cancer separately, compared with IHC/FISH in tissue samples, the sensitivity of dPCR in plasma increases from $37.93 \%$ (11/29) for stage III to $41.67 \%$ (15/36) for stage IV cancer. Recurrent breast cancer patient had an increased sensitivity of $51.61 \%(16 / 31)$. This is consistent with our expectation sensitivity would increase concordantly as tumor burden goes up. On the other hand, specificity decreased from $92.68 \%$ (38/41) for stage III to $86.44 \%$ (51/59) for stage IV cancer. Recurrent breast cancer patient had a specificity of only $67.86 \%(19 / 28)$. This is, in part, due to inter- and intra-tumor heterogeneity. Many patients determined to be negative for HER2 amplification in tissue biopsy could have HER2 positive tumors at other sites, which was detected by the liquid biopsy. This study suggested the necessity of liquid biopsy for HER2 amplification detection and demonstrated digital PCR can be used as a companion diagnostic tool to determine HER2 amplification status. It also suggested that a liquid biopsy should follow a negative result from tissue biopsy to avoid false negative results especially for late-stage breast cancer patients and ones who experienced relapse or became resistant to current therapy. Future studies should focus on therapeutic effects on patients determined to be HER2 positive through liquid biopsy and collecting additional tissue biopsies to identify HER2 positive tumor when the original tissue biopsy and liquid biopsy don't agree.
\end{abstract}

Key words: HER2, liquid biopsy, tissue biopsy, sensitivity, digital PCR

\section{Introduction}

Targeted therapy significantly improves the therapeutic outcome of patients who test positive in the corresponding companion diagnostic tests. For example, breast and gastric cancer patients who tested positive for human epidermal growth factor 2 (HER2) amplification would have improved survival on anti-HER2 therapy [1, 2]. Non-small cell lung cancer patients would benefit from different generations of 
tyrosine kinase inhibitors, depending on the particular mutation identified from epidermal growth factor receptor (EGFR) mutation detection assays [3-6]. Because patients with such mutations would not respond well to regular chemotherapy, it is critically important to avoid false negative test results, so those patients do not miss their opportunity to benefit from targeted therapy [7].

Currently, tissue biopsy is commonly used as a companion diagnostic tool to decide whether a cancer patient would benefit from targeted therapy. However, tissue biopsy results are influenced by inter- and intra-tumor heterogeneity, leading to false negative test results [8-10]. The possibility of having false negative test results is higher for patients with advanced cancer or who have relapsed because they will have bigger and multiple tumors [11]. For these patients, it is difficult to get multiple tissue biopsies to have conclusive results. Additionally, mutations not present in the original biopsy may arise as the cancer progresses [12]. These temporal changes have no way of being detected because tissue biopsies are not repeated, according to clinical standards. Therefore, it is important to supplement traditional tissue biopsy with additional information.

One method that can fortify tissue biopsy results is liquid biopsy. Liquid biopsy detects a variety of cancer related mutations from circulating tumor DNA (ctDNA) $[11,13,14]$. ctDNA contains DNA mutations, epigenetic changes, among other abnormalities which encompasses the genetic topography of tumors. This gives liquid biopsy the potential to circumvent the problems stemming from intra- and inter-tumor heterogeneity. HER2 is a companion diagnostic biomarker for anti-HER2 therapy. In 2016, Otsuhi et al. found that plasma HER2 correlates with tumor size in HER2 positive patients [15]. For these patients, the HER2 copy number status was used to monitor the tumor's response to therapies [15]. HER2 amplification might not have been detected via tissue biopsy for cases with large or multiple tumors, as that method of detection cannot identify all subpopulations of tumor. Additionally, as tumors evolve throughout treatment, HER2 positive tumors may be enriched with regular chemotherapy as they would not respond to the treatment. Acquired HER2 amplification could also happen during regular chemotherapy $[12,14]$. HER2 amplification detection through liquid biopsy allows healthcare providers to prescribe anti-HER2 therapy for patients with false negative test results for liquid biopsy. Additionally, the relative ease of collecting body fluid samples, when compared to the invasive nature of tissue biopsy, allows repeated tests to determine HER2 amplification status during cancer treatment. This is especially important for patients who originally tested negative for HER2 and for the monitoring of therapeutic effects on patients with positive test results treated with anti-HER2 therapy.

HER2 amplification is routinely determined by immunohistochemistry (IHC) or fluorescence in situ hybridization (FISH) on tissue biopsy [16, 17]. Those are tests based on HER2 protein (IHC) or chromosome DNA (FISH) done on formalin-fixed paraffinembedded (FFPE) tissue samples. While these tests are considered the gold standard, IHC has poor reproducibility and is influenced by laboratory errors due to poor standardization; FISH, while being more robust, is limited by spatial heterogeneity because it is performed at high magnification [18-20]. To detect HER2 amplification status in ctDNA, digital PCR (dPCR) is a great candidate technology. dPCR has been designed and developed to improve on the sensitivity of qPCR. Traditional quantitative PCR is only capable of relative quantification [21]. dPCR, on the other hand, achieves absolute quantification through its methodology of partitioning of samples fundamental difference in methodology. For digital PCR, a PCR reaction mixture is partitioned into $20 \mathrm{~K}$ different micro-reactions, resulting in, theoretically, 0 or 1 molecules of target DNA or RNA in each micro-reaction if input amounts of target nucleic acids are proper [22]. After thermocycling, if the target molecule is present, the micro-reaction will have positive fluorescence. Users can count the number of positive micro-reactions to determine how many copies there are in the sample tested. Thus, for HER2 amplification, copy numbers for HER2 and a reference gene will be determined independently through counting the number of micro-reactions. Possion distribution will be used to adjust for number of micro-reactions when more than one copy of HER2 or reference gene may exist. A ratio between HER2 and reference gene can then be calculated accurately through this absolute quantification process; this allows for the detection of small changes in amplification [23].

A previous study found that the coefficient of variance $(\mathrm{CV})$ of plasma HER2 was between $2-3 \%$, and the limit of detection was 2.36 copies per diploid gene [24]. This is significant because if the HER2 copy number is greater than 2.36, $\mathrm{dPCR}$ can detect the copy number variation. This level of sensitivity is especially important for patients who develop HER2 mutations throughout the course of their cancer as the number of HER2 copies would be relatively low. Digital PCR also has an easy to set-up process, fast turnaround time, and easy data interpretation. This allows the technology to be used by more healthcare providers and would benefit a wider variety of patients. 
The goal of our study is to evaluate if digital PCR can satisfy the needs of HER2 liquid biopsy through a clinical lens. The study design includes the following: 1. Compare the positive percent agreement (PPA) and negative percent agreement (NPA) the chip-based digital PCR assay in plasma samples with IHC/FISH results in paired tissue samples; 2 . Confirm the cut off value we set for HER2 amplification based on analytical studies on contrived clinical samples; and 3. Evaluate and conclude if different cut off values/ threshold need to be set for different stages of cancer due to differences with tumor burden. We expect the results to confirm that liquid biopsy can effectively reduce the number of false negative test results from tissue biopsy in late stage or recurrent breast cancer patients due to the influence of intra- and inter-tumor heterogeneity. This suggests the necessity of detecting HER2 amplification by liquid biopsy and that digital PCR can be used to determine the HER2 amplification status.

\section{Materials and methods}

\section{Patient cohort}

The study cohort included 224 patients enrolled from 3 medical centers. Advanced breast cancer was confirmed by common clinical methods. The plasma samples were collected from all patients, and the test results of paired tissue samples from the same patient at the same period were collected. The tissue sample included primary tumors and metastatic lesions. Tumor HER2 status detected by IHC and/or FISH and positive for HER2 defined as $2+$ or $3+$ by IHC or FISH positive. The studies were approved by the Institutional Review Board or Independent Ethics Committee associated with each study center. The general principles of the International Ethical Guidelines for Biomedical Research Involving Human Subjects, the International Conference on Harmonisation guidelines on Good Clinical Practice, and the Declaration of Helsinki were followed. All patients had signed informed consent.

\section{Plasma preparation and cfDNA extraction}

$10 \mathrm{~mL}$ peripheral blood was collected in a PAXgene Blood ccfDNA Tube (Cat No: 768115, Qiagen, Hilden, Germany). The collected whole blood was centrifuged as soon as possible at $1900 \times \mathrm{g}$ for 15 minutes under room temperature. The supernatant was collected and centrifugated again at $1900 \times \mathrm{g}$ for 10 minutes. The supernatant from the second centrifugation was the final plasma which was stored at $-80^{\circ} \mathrm{C}$ until use.

The cfDNA was extracted from the plasma using the Qiagen QIAamp Circulating Nucleic Acid Kit (Cat No: 55114, Qiagen) according to the manufacturer's instructions.

\section{HER2 amplification status analyzed using digital PCR}

The HER2 amplification status was assessed by the ratio of plasma HER2 copy number to reference gene copy number (HER2 ratio), which was performed on a ProFlex 2X Flat PCR system (Cat No: 4484078, Thermo Fisher Scientific, Waltham, MA, USA) using the HER2 Amplification detection kit (Cat No: Q0137365402, Questgenomics, Nanjing, JS, China). A total $14.5 \mu \mathrm{dPCR}$ reaction mixture was prepared with $5.8 \mu \mathrm{l}$ cfDNA sample and RNase-free water (about 5ng cfDNA input), 7.25 $\mu$ l dPCR Master Mix and 1.45 $\mu$ l HER2 amplification detection reaction solution. The dPCR reaction mixture was loaded into chip wells using the Questgenomics Chip Loader and then was sealed and loaded onto ProFlex 2X Flat PCR system according to the manufacturer's instructions. The cycling condition was as follows: $96{ }^{\circ} \mathrm{C}$ for 10 minutes, 39 cycles of $60^{\circ} \mathrm{C}$ for 2 minutes and $98^{\circ} \mathrm{C}$ for 30 seconds, followed by a final extension step at $6{ }^{\circ} \mathrm{C}$ for 2 minutes. The chip images were captured with the Questgenomics Biochip Reader and further analyzed using the Cloud Software from Questgenomics. Negative controls with no DNA were included in each run. A HER2 ratio $\geq 1.3$ was defined as positive HER2 amplification, and a HER2 ratio < 1.3 was defined as negative HER2 amplification.

\section{Statistical analysis}

All statistical analyses were performed using SPSS software (version 19.0) and Graph pad prism 7.04 (GraphPad Software, Inc., La Jolla, CA, USA). A receiver operating characteristic (ROC) curve was used to evaluate the optimal cut-off point in consideration of Youden index and to balance the PPA and NPA and a low false negative rate was chosen as priority. Data are presented as means \pm standard deviation. A $\mathrm{P}$ value less than 0.05 was considered to be statistically significant.

\section{Results}

\section{Patients' Characteristics}

The study included 241 patients from 3 clinical research centers that were screened for HER2 amplification. 17 patients that did not meet the selection criteria were excluded from the study and the samples of 224 remaining patients were analyzed. Of the 224 patients, the median age was 51 years old, $224(100 \%)$ were females, $75(32.89 \%)$ had stage III breast cancer and $149(65.35 \%)$ had stage IV breast cancer. 210 out of 224 patients $(92.11 \%)$ were diagnosed as invasive ductal carcinoma (Table 1). The flow chart of the analyses on these patients is shown 
in Figure 1. Stage III and IV patients are those who have received a diagnosis. Recurrent patients are those who have undergone surgery and have relapsed post-surgery.

Table 1. The characteristics of enrolled patients

\begin{tabular}{lll}
\hline Index & 3 clinical centers (N=224) & Ratio \% \\
\hline Age & & \\
$\mathrm{N}$ & $224(0)$ & $0 \%$ \\
Mean \pm Std & $50.55 \pm 11.04$ & $/$ \\
$\mathrm{M}(\mathrm{Q} 1, \mathrm{Q} 3)$ & $51(43,58)$ & $/$ \\
Min,Max & 29,79 & \\
Age & & $46.88 \%$ \\
$<50$ & 105 & $32.14 \%$ \\
$50-59$ & 72 & $16.96 \%$ \\
$60-69$ & 38 & $4.02 \%$ \\
$>69$ & 9 & $100.00 \%$ \\
Total & 224 & \\
Types of diseases & & $92.11 \%$ \\
Invasive ductal carcinoma & 210 & $1.75 \%$ \\
Invasive lobular carcinoma & 4 & $0.88 \%$ \\
Invasive carcinoma, nonspecific & 2 & $0.44 \%$ \\
Invasive/metastatic carcinoma & 1 & $0.44 \%$ \\
Metastatic/invasive lobular carcinoma & 1 & $0.44 \%$ \\
Medium to high grade ductal carcinoma in situ & 1 & $0.44 \%$ \\
Metastatic breast cancer & 1 & $1.75 \%$ \\
unknown & 4 & \\
Disease stage* & & $32.89 \%$ \\
Stage III & 75 & $65.35 \%$ \\
Stage IV & 149 & $100.00 \%$ \\
Gender & & \\
Female & 224 & \\
\hline *The number of patients listed are from the initial diagnosis. At the time patients \\
participated in this study, 5 stage III and 54 stage IV patients relapsed.
\end{tabular}

We determined the plasma HER2 cut off and calculated the PPA, NPA, and overall compliance rate of HER2 status between digital PCR and IHC/FISH test. The receiver operating characteristic (ROC) curve had been drawn to set the cut-off value of plasma HER2 detection among different clinical stages. Youden index implicates the optimal PPA and NPA by analyzing the tissue HER2 status and plasma HER2 ratio.

\section{Comparison of HER2 amplification detection using digital PCR in blood ctDNA and IHC/FISH in paired tissue biopsy}

According to the results of clinical validation studies on 98 clinical samples using ROC analysis, the cut-off value was determined to be 1.30 of HER2 over reference gene using the absolute quantification capability of digital PCR assay. At this cut-off value, the PPA, NPA, the positive predictive value (PPV), the negative predictive value (NPV) and the concordance were calculated based on the results of plasma detected by digital PCR assay and tissue samples by IHC/FISH. In this study, the prevalence was based off of the sample set and is not a reflection of a population.

For the 224 advanced breast cancer patients enrolled in this study, the PPA between dPCR in plasma and IHC/FISH in tissue samples was $43.75 \%$ (42/96), the specificity was $84.38 \%(108 / 128)$, the PPV was $67.74 \%$ (42/62), the NPV was $66.67 \%$ (108/162), and the concordance was $66.96 \%(150 / 224)$ (Table 2$)$.

Table 2. Concordance of $d P C R$ in plasma and IHC/FISH on tissue HER2 detection in all enrolled breast cancer patients $(\mathrm{N}=224)$

\begin{tabular}{llll}
\hline & \multicolumn{2}{l}{ HER2 with IHC/FISH in tissue } & \multirow{2}{*}{ Total } \\
\cline { 2 - 3 } & Positive & Negative & \\
\cline { 1 - 2 } ctDNA HER2 with dPCR in plasma & & 62 \\
\hline Positive & 42 & 20 & 162 \\
Negative & 54 & 108 & 224 \\
Total & 96 & 128 & \\
\hline
\end{tabular}

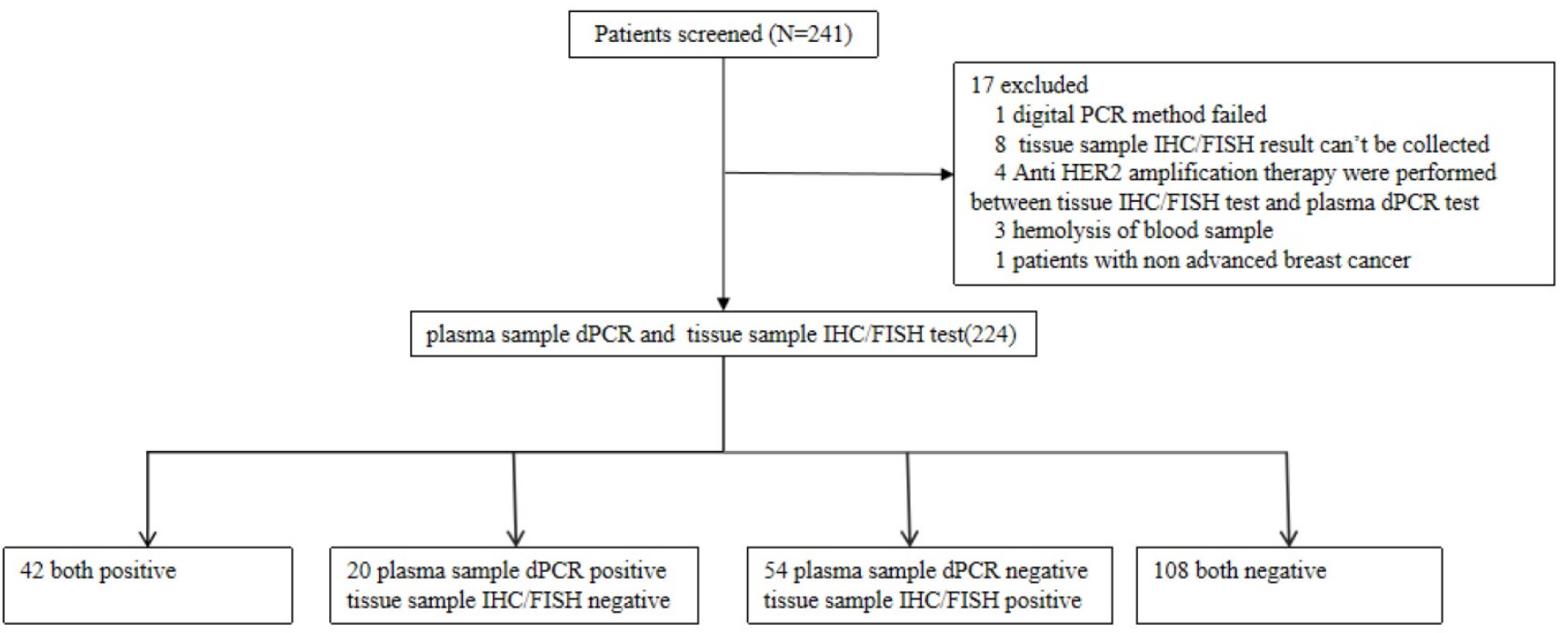

Figure 1. Flow chart of the analyses on enrolled patients. 


\section{Positive percent agreement, negative percent agreement and overall concordance are different for different stage cancer}

We further analyzed the consistency of digital PCR in plasma samples and IHC/FISH in tissue samples in patients with stage III, IV and recurrent cancer respectively. We have found that for the 70 stage III breast cancer patients, the PPA between dPCR in plasma and IHC/FISH in tissue samples was $37.93 \%(11 / 29)$, the NPA was $92.68 \%(38 / 41)$, the PPV was $78.57 \%(11 / 14)$, the NPV was $67.86 \%(38 / 56)$ and the concordance was $70.00 \%(49 / 70)$ (Table 3$)$.

For the 95 stage IV breast cancer patients, the PPA between dPCR in plasma and IHC/FISH in tissue samples was $41.67 \%(15 / 36)$, the NPA was $86.44 \%(51 / 59)$, the PPV was $65.22 \%(15 / 23)$, the NPV was $70.83 \%(51 / 72)$, and the concordance was $69.47 \%$ (66/95) (Table 4).

For the 59 patients with recurrent breast cancer, the PPA between dPCR in plasma and IHC/FISH in tissue samples was $51.61 \%(16 / 31)$, the NPA was $67.86 \%(19 / 28)$, the PPV was $64.00 \%(16 / 25)$, the NPV was $55.88 \%(19 / 34)$, and the concordance was $59.32 \%$ $(35 / 59)$ (Table 5).

The PPA increases from $37.93 \%(11 / 29)$ for stage III to $41.67 \%(15 / 36)$ for stage IV cancer. And recurrent breast cancer patients had an increased PPA of $51.61 \%(16 / 31)$. The results showed that the PPA would increase concordantly as tumor burden increases. On the other hand, NPA decreased from $92.68 \%(38 / 41)$ for stage III to $86.44 \%(51 / 59)$ for stage IV cancer. Recurrent breast cancer patients had a NPA of only $67.86 \%(19 / 28)$. This suggests that the interand intra-tumor heterogeneity gives rise to this result, which also suggests the necessity of liquid biopsy for HER2 amplification detection.

Table 3. Concordance of $\mathrm{dPCR}$ in plasma and IHC/FISH on tissue HER2 detection in stage III breast cancer patients $(\mathrm{N}=70)$

\begin{tabular}{|c|c|c|c|}
\hline & \multicolumn{2}{|c|}{ HER2 with IHC/FISH in tissue } & \multirow[t]{2}{*}{ Total } \\
\hline & Positive & Negative & \\
\hline \multicolumn{4}{|c|}{ ctDNA HER2 with dPCR in plasma } \\
\hline Positive & 11 & 3 & 14 \\
\hline Negative & 18 & 38 & 56 \\
\hline Total & 29 & 41 & 70 \\
\hline
\end{tabular}

Table 4. Concordance of $\mathrm{dPCR}$ in plasma and IHC/FISH on tissue HER2 detection in stage IV breast cancer patients $(\mathrm{N}=95)$

\begin{tabular}{llll}
\hline & \multicolumn{2}{c}{ HER2 with IHC/FISH in tissue } & Total \\
\cline { 2 - 3 } & \multicolumn{2}{c}{ Positive } & Negative \\
\hline ctDNA HER2 & with dPCR in plasma & & \\
\hline Positive & 15 & 8 & 23 \\
Negative & 21 & 51 & 72 \\
Total & 36 & 59 & 95 \\
\hline
\end{tabular}

Table 5. Concordance of $\mathrm{dPCR}$ in plasma and IHC/FISH on tissue HER2 detection in recurrent breast cancer patients $(\mathrm{N}=59)$

\begin{tabular}{llll}
\hline & \multicolumn{2}{l}{ HER2 with IHC/FISH in tissue } & \multirow{2}{*}{ Total } \\
\cline { 2 - 3 } \multicolumn{1}{c}{ Positive } & Negative & \\
\cline { 1 - 2 } ctDNA HER2 & with dPCR in plasma & 9 & 25 \\
\hline Positive & 16 & 19 & 34 \\
Negative & 15 & 28 & 59 \\
Total & 31 & & \\
\hline
\end{tabular}

\section{ROC analysis to determine cut-off value for different stage cancer}

For the $70 \mathrm{dPCR}$ in plasma samples of stage III breast cancer patients, the IHC/FISH in paired FFPE samples as reference, the cut-off value was recommended to be 1.28 with the maximum Youden index. The area under the ROC curve (AUC) was 0.6501 with the PPA and NPA of $37.93 \%(11 / 29)$ and $92.68 \%(38 / 41)$, respectively. The concordance between dPCR in plasma samples and IHC/FISH in tissue samples for stage III breast cancer was $70.00 \%$ $(49 / 70)$ shown in Table 6.

When the cutoff was 1.30, the PPA and NPA of the detection results of stage III breast cancer patients were as same as that when cutoff was 1.28 (Table 3).

For the $95 \mathrm{dPCR}$ in plasma samples of stage IV breast cancer patients, the IHC/FISH in paired FFPE samples as reference, the cut-off value was recommended at 1.58 with the maximum Youden index. The AUC was 0.7079 with the PPA and NPA of $36.11 \%(13 / 36)$ and $94.92 \%(56 / 59)$, respectively. The concordance between dPCR in plasma samples and IHC/FISH in tissue samples for stage IV breast cancer was $72.63 \%(69 / 95)$ shown in Table 7.

Table 6. Concordance of $\mathrm{dPCR}$ in plasma and IHC/FISH on tissue HER2 detection in stage III breast cancer patients $(\mathrm{N}=70)$ with cut off value of 1.28

\begin{tabular}{|c|c|c|c|}
\hline & \multicolumn{2}{|c|}{ HER2 with IHC/FISH in tissue } & \multirow[t]{2}{*}{ Total } \\
\hline & Positive & Negative & \\
\hline \multicolumn{4}{|c|}{ ctDNA HER2 with dPCR in plasma } \\
\hline Positive & 11 & 3 & 14 \\
\hline Negative & 18 & 38 & 56 \\
\hline Total & 29 & 41 & 70 \\
\hline
\end{tabular}

Table 7. Concordance of $\mathrm{dPCR}$ in plasma and IHC/FISH on tissue HER2 detection in stage IV breast cancer patients $(\mathrm{N}=95)$ with cut off value of 1.58

\begin{tabular}{llll}
\hline & \multicolumn{2}{c}{ HER2 with IHC/FISH in tissue } & Total \\
\cline { 2 - 3 } & \multicolumn{2}{c}{ Positive } & Negative \\
\cline { 1 - 2 } ctDNA HER2 & with dPCR in plasma & 3 & 16 \\
\hline Positive & 13 & 56 & 79 \\
Negative & 23 & 59 & 95 \\
Total & 36 & & \\
\hline
\end{tabular}

The PPA and NPA were $41.67 \%(15 / 36)$ and $86.44 \%(19 / 28)$ respectively when evaluated at the threshold at 1.30, The concordance between dPCR in 
plasma and IHC/FISH on FFPE tissue samples for advanced breast was $69.47 \%$ (35/59) shown in Table 4.

For the $165 \mathrm{dPCR}$ in plasma samples of stage III and stage IV breast cancer patients, the IHC/FISH in paired FFPE samples as the reference, the cut-off value was recommended at 1.33 with the maximum Youden index. The AUC was 0.6839 with the PPA and NPA of $38.46 \% \quad(25 / 65)$ and $90.00 \% \quad(90 / 100)$, respectively. The concordance between $\mathrm{dPCR}$ in plasma samples and IHC/FISH in tissue samples for advanced breast cancer was $69.70 \%(115 / 165)$ shown in Table 8.

The PPA and NPA were $40.00 \%(26 / 65)$ and $89.00 \%(89 / 100)$ respectively when evaluated at the threshold at 1.30, The concordance between dPCR in plasma and IHC/FISH on FFPE tissue samples for advanced breast cancer was $69.70 \%(115 / 165)$ shown in Table 9.

For the $59 \mathrm{dPCR}$ in plasma samples of recurrent breast cancer, the IHC/FISH in paired FFPE samples as reference, the cut-off value was recommended at 1.56 with the maximum Youden index. The AUC was 0.6285 with the PPA and NPA of $45.16 \%$ (14/31) and $89.29 \%(25 / 28)$ respectively. The concordance between dPCR in plasma samples and IHC/FISH in tissue samples for recurrent advanced breast cancer was $66.10 \%(39 / 59)$ shown in Table 10.

However, the PPA and NPA were 51.61\% $(16 / 31)$ and $67.86 \%(19 / 28)$ when evaluated at the threshold at 1.30, respectively. The concordance between dPCR in plasma and IHC/FISH on FFPE tissue samples for recurrent advanced breast cancer was 59.32\% (35/59) shown in Table 5.

Table 8. Concordance of $\mathrm{dPCR}$ in plasma and IHC/FISH on tissue HER2 detection in stage III and IV breast cancer patients $(\mathrm{N}=165)$ with cut off value at 1.33

\begin{tabular}{lcll}
\hline & \multicolumn{2}{c}{ HER2 with IHC/FISH in tissue } & Total \\
\cline { 2 - 3 } & Positive & Negative & \\
\cline { 1 - 2 } ctDNA HER2 & with dPCR in plasma & 10 & 35 \\
\hline Positive & 25 & 90 & 130 \\
Negative & 40 & 100 & 165 \\
Total & 65 &
\end{tabular}

Table 9. Concordance of $d P C R$ in plasma and IHC/FISH on tissue HER2 detection in stage III and IV breast cancer patients $(\mathrm{N}=165)$ with cut off value at 1.30

\begin{tabular}{llll}
\hline & \multicolumn{2}{l}{ HER2 with IHC/FISH in tissue } & Total \\
\cline { 2 - 3 } \multicolumn{2}{l}{ Positive } & Negative & \\
\hline ctDNA HER2 & with dPCR in plasma & & 37 \\
\hline Positive & 26 & 11 & 128 \\
Negative & 39 & 89 & 165 \\
Total & 65 & 100 & \\
\hline
\end{tabular}

Table 10. Concordance of $\mathrm{APCR}$ in plasma and IHC/FISH on tissue HER2 detection in recurrent breast cancer patients $(\mathrm{N}=59)$ with cut off value at 1.56

\begin{tabular}{llll}
\hline & \multicolumn{2}{c}{ HER2 with IHC/FISH in tissue } & Total \\
\cline { 2 - 3 } & Positive & Negative & \\
\cline { 1 - 3 } ctDNA HER2 & with dPCR in plasma & & 17 \\
\hline Positive & 14 & 3 & 42 \\
Negative & 17 & 25 & 59 \\
Total & 31 & 28 & \\
\hline
\end{tabular}

\section{Discussion}

We have confirmed the cut off value for the digital PCR based assay to be at 1.30 for plasma samples. The PPA, NPA, and overall concordance of HER2 amplification status between digital PCR plasma samples and IHC/FISH paired tissue samples were calculated at this cut off value. An increase in PPA became evident as it increased from 37.93\% $(11 / 29)$ for stage III cancer patients to $41.67 \%(15 / 36)$ for stage IV and $51.61 \%(16 / 31)$ for patients who relapsed after surgery. This gradient is due to an increase in tumor burden, which would include an increase in number of tumors and the cumulated mass of multiple tumors combined. Therefore, the PPA is higher in late-stage cancer.

NPA, on the other hand, reflects an opposite trend of PPA, which started at $92.68 \%(38 / 41)$ for stage III breast cancer patients to $86.44 \%(51 / 59)$ for stage IV and $67.86 \%(19 / 28)$ for relapsed patients. This decrease in NPA is significantly higher than the increase in PPA for the same group of samples. This suggests that there are more factors contributing to it than an increase in tumor burden. This result also suggests that the decrease in NPA could not be due to false positive test results. On the other hand, this observed difference of NPA may be due to varying degrees of inter- and intra-tumor heterogeneity between the stages of cancer. Tissue biopsy normally takes one tissue sample from one tumor. If there are multiple tumors in the body, they would not be detected. Additionally, tissue samples do not reflect the mosaic of mutations present throughout a single tumor. It is very possible that if a small tissue sample is taken, HER2 amplification will not be detected even if amplification may exist elsewhere within that tumor due to spatial heterogeneity.

Besides the spatial heterogeneity, temporal heterogeneity was also reported by a plethora of studies $[9,15,24,25]$. After relapse or when patients develop resistance to the current chemotherapy, HER2 amplification may increase. That is when an additional HER2 liquid biopsy should be done. It is logical to assume that a HER2 liquid biopsy assay based on dPCR would identify HER2 positive patients even if they may have a negative result from tissue biopsy, and this is especially relevant for late-stage 
cancer patients. Therefore, liquid biopsy is necessary to help avoid many false negative results for HER2 amplification.

There have been discussions with oncologists during the process of the clinical study whether different cut off values should be arranged for different cancer stages. Having investigated data in detail, we have concluded that a single cut off value should be set at 1.30. As shown in the result session, although we have seen better PPA, NPA, and overall concordance if a different cut off value is selected based on the optimal Youden index, we believe it could be misleading as the discrepancy between test results from plasma and paired tissue samples may result from spatial and temporal tumor heterogeneity. This suggests that the reference method for tissue biopsy had a systematic error, and therefore may not be the optimal reference gold-standard for a ROC analysis, especially when it is expected to have a higher false negative rate due to increased tumor size and number of tumors for relapse cancer patients. Additionally, although increasing the cutoff value results in higher specificity, the degree to which the PPA is lowered makes increasing the cutoff value inadvisable. With the higher cutoff value, true positive datapoints are lost. This is especially harmful to recurrence and metastasis patients as they typically have higher degrees of inter- and intra-tumor heterogeneity and to patients with acquired HER2 positive status as they have lower copy numbers. The number of false negative results would be high and would misinform healthcare professionals leading to ill-informed treatment plans.

Liquid biopsy as a companion diagnostic tool is incredibly important for HER2 positive patients. Many patients who are negative for HER2 via tissue biopsy may be found positive with liquid biopsy; this is confirmed with a previous study which found that detection of the EGFR T790M mutation is comparable between liquid and tissue biopsy [26]. This technology is especially important for patients who have relapsed, as a second tissue biopsy is typically not conducted. These patients would greatly benefit from continual monitoring. Liquid biopsy is the best method, out of current technologies, for this type of monitoring since the collection of samples is easier and less invasive than traditional tissue biopsy. For patients who are found to be negative with tissue but positive with liquid biopsy, another tissue biopsy should be taken. This is corroborated with National Comprehensive Cancer Network guidelines which state that if a patient is found negative via liquid biopsy, a tissue biopsy test should be ordered, if possible [27].

In conclusion, the newly developed digital PCR detection has high specificity and cost-effectiveness, which can be an effective tool to detect plasma HER2 status.

However, our study still has some limitations. Although it can be seen from the results that the tissue IHC/FISH results of recurrence or metastasis breast cancer patients should be false negative due to the inter or intra tumor heterogeneity, we have not been able to collect additional tissues to confirm that. Therefore, our future studies should focus on therapeutic effects on patients determined to be HER2 positive through liquid biopsy and collecting additional tissue biopsies to identify HER2 positive tumor when the original tissue and liquid biopsy don't agree.

\section{Abbreviations}

AUC: area under the (ROC) curve; ctDNA: circulating tumor DNA; $\mathrm{CV}$ : coefficient of variance; dPCR: digital polymerase chain reaction; EGFR: epidermal growth factor receptor; FFPE: formalinfixed paraffin-embedded; FISH: fluorescence in situ hybridization; HER2: human epidermal growth factor 2; IHC: immunohistochemistry; NPA: negative percent agreement; NPV: negative predictive value; PCR: polymerase chain reaction; PPA: positive percent agreement; PPV: positive predictive value; ROC: receiver operating characteristic.

\section{Acknowledgements}

The authors would like to thank all doctors of the Department of breast surgery of Fudan University Shanghai Cancer Center, all doctors of the Department of oncology of National Cancer Center/National Clinical Research Center for Cancer/Cancer Hospital and all doctors of the Department of oncology of Affiliated Hospital of Jiangnan University for providing all the necessary information required for this study.

\section{Funding}

The work was financially supported by grants from the National Natural Science Foundation of China (\#81772808, \#81772774, \#82072876) and Shanghai "Rising Stars of Medical Talents" Youth Development Program (SHWRS2020_087).

\section{Ethics Statement}

The studies were approved by the Institutional Review Board or Independent Ethics Committee associated with each study center. The general principles of the International Ethical Guidelines for Biomedical Research Involving Human Subjects, the International Conference on Harmonisation guidelines on Good Clinical Practice, and the 
Declaration of Helsinki were followed. All patients had signed informed consent.

\section{Competing Interests}

The authors have declared that no competing interest exists.

\section{References}

1. Patel A, Unni N, and Peng Y. The changing paradigm for the treatment of HER2-positive breast cancer. Cancers (Basel). 2020; 12: 2081

2. Swain SM, Baselga J, Kim SB, et al. Pertuzumab, trastuzumab, and docetaxel in HER2-positive metastatic breast cancer. N Engl J Med. 2015; 372: 724-34.

3. Lee CK, Wu YL, Ding PN, et al. Impact of specific epidermal growth factor receptor (EGFR) mutations and clinical characteristics on outcomes after treatment with EGFR tyrosine kinase inhibitors versus chemotherapy in EGFR-mutant lung cancer: A meta-analysis. J Clin Oncl. 2015; 33: 1958-65.

4. Sequsit LV, Yang JC, Yamamoto N, et al. Phase III study of afatinib or cisplatin plus pemetrexed in patients with metastatic lung adenocarcinoma with EGFR mutations. J Clin Oncol. 2013; 31: 3327-34.

5. Mitsudomi T, Morita S, Yatabe Y et al. Gefitinib versus cisplatin plus docetaxel in patients with non-small-cell lung cancer harbouring mutations of the epidermal growth factor receptor (WJTOG3405): An open label, randomised phase 3 trial. Lancet Oncl. 2010; 11: 121-28.

6. Rosell R, Carcereny E, Gervais R, et al. Erlotinib versus standard chemotherapy as first-line treatment for European patients with advanced EGFR-mutation-positive non-small-cell lung cancer (EURTAC): A multicentre, open-label, randomised phase 3 trial. Lancet Oncol. 2012; 13: 239-46.

7. Noordhuis MG, Eijsink JJH, ten Hoor KA, et al. Expression of epidermal growth factor receptor (EGFR) and activated EGFR predict poor response to (chemo)radiation and survival in cervical cancer. Clin Cancer Res. 2009; 15: 7389-97.

8. Gerlinger $\mathrm{M}$, Rowan $\mathrm{AJ}$, Horswell $\mathrm{S}$, et al. Intratumor heterogeneity and branched evolution revealed by multiregion sequencing. N Engl J Med. 2012; 366: 883-92.

9. Caswell-Jin JL, McNamara K, Reiter JG, et al. Clonal replacement and heterogeneity in breast tumors treated with neoadjuvant HER2-targeted therapy. Nat Commun. 2019; 10: 657.

10. Bedard PL, Hansen AR, Ratain MJ, Siu LL. Tumor heterogeneity in the clinic. Nature. 2013; 501: 355-64.

11. Kim B, Nam SK, Seo SH, et al. Comparative analysis of HER2 copy number between plasma and tissue samples in gastric cancer using droplet digital PCR. Sci Rep. 2020; 10: 4177.

12. Joung JG, Bae JS, Kim SC, et al. Genomic characterization and comparison of multi-regional and pooled tumor biopsy specimen. PLoS One. 2016; 11: e0152574.

13. Keller L, Belloum Y, Wikman H, and Pantel K. Clinical relevance of bloodbased ctDNA analysis: Mutation detection and beyond. Br J Cancer. 2021; 124 : $345-58$

14. Fiala C, Diamandis EP. Utility of circulating tumor DNA in cancer diagnostic with emphasis on early detection. BMC Medicine. 2018; 16: 166.

15. Shoda K, Ichikawa D, Fujita Y, et al. Monitoring the HER2 copy number status in circulating tumor DNA by droplet digital PCR in patients with gastric cancer. Gastric Cancer. 2017; 20: 126-35.

16. [Internet] National Comprehensive Cancer Network. NCCN Guidelines for Patients: Breast Cancer, Metastatic. Effective date 2020. http://www.nccn. org/patients.

17. Cuadros $\mathrm{M}$ and Villegas R. Systematic review of HER2 breast cancer testing. Appl Immunohistochem Mol Morphol. 2009; 17: 1-7.

18. Hick DG Schiffhauer L. Standardized assessment of HER2 status in breast cancer by immunohistochemistry. Laboratory Medicine. 2011; 42: 459-67.

19. Furrer D, Sanschagrin F, Jacob S, Diorio C. Advantages and disadvantages of technologies for HER2 testing in breast cancer specimens. Am J Clin Pathol. 2015; 144: 686-703.

20. Bilous M, Dowsett M, Hanna W, et al. Current perspectives on HER2 testing: A review of national testing guidelines. Mod Pathol. 2003; 16: 173-82.

21. VanGuilder HD, Vrana KE, and Freeman WM. Twenty-five years of quantitative PCR for gene expression analysis. BioTechniques. 2008; 44: $619-26$.

22. Belgrader P, Tanner SC, Regan JF, et al. Droplet Digital PCR Measurement of HER2 Copy Number Alteration in Formalin-Fixed Paraffin-Embedded Breast Carcinoma Tissue. Clin Chem. 2013; 59: 991-4.

23. Gevensleben $\mathrm{H}$, Garcia-Murillas I Graeser MK, et al. Noninvasive detection of HER2 amplification with plasma DNA digital PCR. Clin Cancer Res. 2013; 19: 3276-84

24. Zhou $R$, Yuan $P$, Zhang $L$, et al. Using digital PCR to detect HER2 amplification in breast and gastric cancer patients. Frontiers in Laboratory Medicine. 2018; 2: 102-8

25. Keup C, Suryaprakash V, Storbeck M, et al. Longitudinal multi-parametric liquid biopsy approach identifies unique features of circulating tumor cell, extracellular vesicle, and cell-free DNA characterization for disease monitoring in metastatic breast cancer patients. Cells. 2021; 10: 212.

26. Zhou R, Cai Y, Li Z, et al. A digital PCR assay development to detect EGFR T790M mutation in NSCLC patients. Frontiers in Laboratory Medicine. 2018; 2: 89-96.

27. [Internet] National Comprehensive Cancer Network. NCCN Guidelines for Patients: Non-Small Cell Lung Cancer, Metastatic. Effective date 2021. http://www.nccn.org/patients. 